\title{
Nosocomial Fever in General Medical Wards: A Prospective Cohort Study of Clinical Characteristics and Outcomes
}

\author{
Parita Dankul' \\ Khemajira Karaketklang' \\ Anupop Jitmuang ${ }^{2}$ \\ 'Department of Medicine, Faculty of \\ Medicine Siriraj Hospital, Mahidol \\ University, Bangkok, Thailand; '² Division \\ of Infectious Diseases and Tropical \\ Medicine, Department of Medicine, \\ Faculty of Medicine Siriraj Hospital, \\ Mahidol University, Bangkok, Thailand
}

Purpose: Nosocomial fever (NF) is a common sign of healthcare-associated infection; however, infection is not always followed up. We studied the etiology, clinical characteristics, and outcomes of nosocomial fever in hospitalized patients.

Patients and Methods: Between October 2019 and December 2020, we enrolled subjects from general medical wards who developed fever $\geq 48$ hours after hospital admission or who were admitted with fever, defervesced, and then developed a fever $\geq 7$ days later that was unrelated to the cause for admission. Subjects with NF underwent a comprehensive clinical evaluation and laboratory investigations.

Results: Eighty-six cases of NF were identified and completely followed, the mean age was 69.29 years, and 35 were male. Fifty-seven were from infectious etiologies, 28 from noninfectious etiologies, and one case was unable to be determined. Hospital-associated pneumonia $(47.4 \%)$ and urinary tract infection $(22.8 \%)$ were the most common infectious causes, and malignancy (17.8\%) and large hematoma (14.3\%) were the most common non-infectious causes. The median day of onset of NF following hospitalization was 12 (4.7-21.2) days. Acute physiology and chronic health evaluation II (APACHE II) score (14.70 vs 11.97, p = 0.02), sequential organ failure assessment (SOFA) scores ( 4 vs $2, \mathrm{p}<0.01)$, pertinent clinical findings $(82.5 \%$ vs $42.9 \%, p<0.01)$, blood urea nitrogen (BUN) $(37.30$ vs $21.10, p=0.03)$ and creatinine ( 1.41 vs $0.97, \mathrm{p}=0.05)$ levels, and abnormal chest radiography $(45.6 \% \mathrm{vs} 3.6 \%, \mathrm{p}<0.01)$ had significant differences between infectious and non-infectious etiologies. Twenty-three subjects (26.7\%) died. The presence of end-stage renal disease (ESRD) [OR 19.49 (1.77-214.18), $\mathrm{p}=$ 0.015 ], SOFA score $>6$ [OR $5.18(1.04-25.90), \mathrm{p}=0.045$ ], and abnormal chest radiography [OR $3.45(1.16-10.29), \mathrm{p}=0.026]$ were significantly associated with mortality.

Conclusion: Nosocomial infections, malignancy, and hematoma were the leading causes of NF. Severity scores, clinical findings, renal function tests, and chest radiography were distinguishing features between infectious and non-infectious etiologies. ESRD, high SOFA scores, and abnormal chest radiography were associated with mortality.

Keywords: nosocomial fever, infectious etiology, non-infectious etiology, outcomes

\section{Introduction}

Nosocomial fever (NF) is a new fever $\geq 48$ hours after hospital admission. ${ }^{1}$ The prevalence of nosocomial fever reported in general medical wards varies from 2$36 \%$ based on the case definition, study populations, study periods, and hospital settings. ${ }^{2-5}$ Generally, fever originates from an interplay between proinflammatory cytokines, the hypothalamus, and cellular and end-organ systems, that are triggered by microorganisms, exogenous pyrogens, tissue inflammation, ischemia, or injury. ${ }^{6}$ 
Thus, nosocomial fever may also have a non-infectious etiology. Among hospitalized patients with NF, $40-70 \%$ of cases are from infectious etiologies, particularly hospitalassociated infections, which are usually associated with antimicrobial resistant organisms leading to prolonged hospital stay, increased costs, and high fatality, ${ }^{7,8}$ while $15-50 \%$ originate from non-infectious etiologies such as malignancies, procedure- or surgery- related conditions, or organ ischemia. In $10-30 \%$ of cases, the etiology cannot be determined. ${ }^{1-3,5,9-11}$ A 1-year retrospective, crosssectional study of patients in general medical wards in Siriraj Hospital reported that $7.5 \%$ of patients developed $\mathrm{NF}$, and $77 \%$ had an infectious etiology, $8 \%$ had a noninfectious cause, and $15 \%$ etiology not determined. ${ }^{12}$ However, clinical outcomes among patients with NF still have been unknown. ${ }^{12}$

$\mathrm{NF}$ is a common condition that requires a review of clinical data since admission, repeated physical examinations, additional laboratory investigations and radiological studies, and specialist consultation for patients with unclear etiology. Data on the causes of NF in Thailand are limited. Thus, we undertook a study to determine the etiology, clinical manifestations, diagnostic investigation, management, and patient outcomes, and factors associated with in-hospital mortality in patients with NF.

\section{Materials and Methods}

\section{Study Design and Study Populations}

A single-center prospective cohort study at Siriraj Hospital, Mahidol University, Bangkok, Thailand was conducted from October 2019 to December 2020. Hospitalized subjects with NF admitted to the general medical wards were enrolled. NF has a variety of acceptable criteria based on patient's age, patient immune status, and severity of illness. ${ }^{5,9,13}$ We defined NF as 1) a new onset of fever $\geq 38.0{ }^{\circ} \mathrm{C}$ by oral temperature or $\geq 38.3{ }^{\circ} \mathrm{C}$ by axillary temperature on at least two consecutive occasions in a subject that had been hospitalized for $\geq 48$ hours, or 2) a hospitalized subject admitted with fever who defervesces but then develops a new fever $\geq 7$ days later that is unrelated to the initial cause of hospital admission. We exclude subjects with febrile neutropenia because our previous study demonstrated etiology, clinical course and treatment outcomes of these subjects were highly different compared to non-neutropenic patients with NF. ${ }^{14}$ Subjects who did not consent to participate in the study were also excluded.
Subjects were assessed to confirm eligibility and obtained informed consent. After enrollment, a comprehensive clinical evaluation and diagnostic investigation was conducted within 72 hours after the onset of fever. The comprehensive clinical evaluation and diagnostic investigation applies an integrated approach that includes a thorough review of the medical history, repeated medical examinations, extensive laboratory investigations and radiological studies, tissues biopsy where indicated, and specialist consultation (Supplementary Table 1). All subjects were followed until discharge or death. Baseline demographic data, patient immunity, living status, cause of hospitalization, the onset date of NF, medical procedures prior to the onset of NF, clinical characteristics of NF, and the results of diagnostic investigations and laboratory testing were recorded. Clinical management, treatment outcomes, and discharge status were also recorded. The etiology of NF was based on a review of all clinical data and the consensus opinion of the investigators.

\section{Definitions}

We used the US CDC/NHSN surveillance definition for healthcare-associated nosocomial infection. ${ }^{15}$ Noninfectious NF included malignancy, organ ischemia or infarction, inflammatory disease, non-infectious arthritis, hypersensitivity or drug reaction, procedure-related, hemorrhage, venous thrombosis, and other possible causes. ${ }^{1,5,9}$ An immunocompromised state was defined as subjects who received systemic corticosteroids, immunosuppressive agents, or chemotherapies, or subjects who had HIV-infection, malignancy, or organ transplantation. Appropriate antimicrobial treatment was an administration of antimicrobial agent(s) in regard to clinical infection, culture, and drug susceptibility testing results. If the etiology could not be determined, it was defined as etiology unknown. Treatment outcomes were classified into, 1) Cure; No fever or a reduction of body temperature less than $37.5^{\circ} \mathrm{C}$ from the baseline fever for more than 72 hours with overall clinical improvement, 2) Partial response; a partial reduction of body temperature from the baseline fever for more than 72 hours or overall clinical improvement, 3) Failure; no reduction or an increase of body temperature from the baseline fever with no overall clinical improvement and/or development of concurrent complications, 4) Relapse; a new onset of NF after the previous onset was cured or improved for more than 72 
hours, and 5) Death; the subject died during the hospitalization.

\section{Statistical Analysis}

A previous study from our hospital reported that the incidence of infectious, non-infectious, and unknown etiologies of $\mathrm{NF}$ in general medical wards were $74 \%, 13.5 \%$ and $12.5 \%$, respectively. ${ }^{12}$ By estimating proportion of one group formula, the sample size has to be 74 subjects. We recorded and analyzed the data with PASW Statistics for Windows (version 16.0; SPSS Inc., Chicago, IL, USA). Continuous variables were presented as mean with standard deviation, medians and percentiles. Categorical variables were summarized as counts and percentages. Inferential statistics were analyzed using Chi square test or Fisher's exact test. Quantitative statistics were analyzed with independent $T$-test for normal distribution data and Mann-Whitney $U$-test for data without normal distribution. Variables that were significantly associated with in-hospital mortality on univariate analysis $(\mathrm{p}<0.1)$ were included in a multivariate logistic regression model. A p-value less than 0.05 was defined as statistically significant.

\section{Results}

Of 100 enrolled subjects, we included total 86 subjects who had complete evaluation and follow-up throughout the study period. Fourteen subjects were excluded from the complete evaluation because they were receiving palliative care. The mean age of the subjects was $69.29 \pm 17.5$ years, and $35(40.7 \%)$ were male (Table 1). Eighteen $(20.9 \%)$ subjects were immunocompromised. Prior to admission, $36(41.9 \%)$ subjects had been dependent on other persons for their daily care. The Median Charlson Comorbidity Index was 5 (3-6). The primary reason for hospitalization was non-infectious in $68.6 \%$ of cases, such as coronary artery disease, congestive heart failure, cerebrovascular disease, decompensated liver disease, and exacerbation of pulmonary disease, and renal failure. Forty-seven $(54.7 \%)$ of subjects had received antimicrobial therapy prior to the onset of NF, an administration of antimicrobial agent(s) for subjects before the onset of NF was judged by attending physicians during the initial course of hospitalization, and subjects had undergone medical procedures prior to the onset of NF including urinary catheterization 59 (68.6\%), nasogastric tubing 47 (54.7\%), endotracheal intubation 25 (29.1\%), and intravascular catheterization $14(16.3 \%)$.
Table I Baseline Demographic Data, Clinical Characteristics, and Treatment Outcomes of All Subjects Who Developed Nosocomial Fever

\begin{tabular}{|c|c|}
\hline Characteristics & Total $(n=86)$ \\
\hline Male (\%) & $35(40.7)$ \\
\hline Mean age (SD), years & $69.29(17.5)$ \\
\hline Mean BMI (SD), kg/m² & $21.29(4.9)$ \\
\hline \multicolumn{2}{|l|}{ Underlying disease (\%) } \\
\hline Hypertension & $51(59.3)$ \\
\hline Dyslipidemia & $31(36)$ \\
\hline Diabetes mellitus & $30(34.9)$ \\
\hline CKD or ESRD & $22(25.6)$ \\
\hline Cerebrovascular disease & $21(24.4)$ \\
\hline Coronary arterial disease or arrhythmia & $16(18.6)$ \\
\hline Autoimmune diseases & $13(15.1)$ \\
\hline Asthma or COPD & $7(8.1)$ \\
\hline Chronic liver disease & $7(8.1)$ \\
\hline HIV infection & $\mathrm{I}(\mathrm{I} .2)$ \\
\hline Organ transplant recipient & $\mathrm{I}(\mathrm{l} .2)$ \\
\hline Immunocompromised state (\%) & 18 (20.9) \\
\hline Living in dependence status (\%) & $36(41.9)$ \\
\hline Median Charlson Comorbidity Index (P25-P75) & $5(3-6)$ \\
\hline \multicolumn{2}{|l|}{ Cause of hospitalization (\%) } \\
\hline Non-infection related & $59(68.6)$ \\
\hline Infection related & $27(31.4)$ \\
\hline Receiving antimicrobial(s) prior to NF diagnosis (\%) ${ }^{\mathrm{a}}$ & $47(54.7)$ \\
\hline \multicolumn{2}{|l|}{ Medical procedures prior to NF diagnosis (\%) } \\
\hline Urinary catheterization & $59(68.6)$ \\
\hline Nasogastric tubing & $47(54.7)$ \\
\hline Endotracheal intubation & $25(29.1)$ \\
\hline Intravascular catheterization & $14(16.3)$ \\
\hline Percutaneous drainage & $2(2.3)$ \\
\hline Recent surgery (\%) & $14(16.3)$ \\
\hline \multicolumn{2}{|l|}{ Clinical characteristics of nosocomial fever } \\
\hline Median onset (P25-P75), days & $12(4.7-21.2)$ \\
\hline Mean body temperature $(\mathrm{SD}),{ }^{\circ} \mathrm{C}$ & $38.96(0.55)$ \\
\hline \multicolumn{2}{|l|}{ Managements (\%) } \\
\hline Receiving antimicrobial treatment & $77(89.5)$ \\
\hline Appropriate antimicrobial treatment & $39(50.6)$ \\
\hline Surgical treatment & $4(14.3)$ \\
\hline Blood transfusion therapy & $27(31.4)$ \\
\hline Median LOS (P25-P75), days & $40(24-62)$ \\
\hline \multicolumn{2}{|l|}{ Treatment outcomes (\%) } \\
\hline Cure & $48(55.8)$ \\
\hline Death & $23(26.7)$ \\
\hline Partially response & II (I2.8) \\
\hline Failure & $2(2.3)$ \\
\hline Relapse & $\mathrm{I}(1.2)$ \\
\hline Not determined & $\mathrm{I}(1.2)$ \\
\hline
\end{tabular}

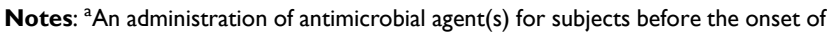
NF was judged by attending physicians during the initial course of hospitalization. Abbreviations: BMI, body mass index; CKD, chronic kidney disease; COPD, chronic obstructive pulmonary disease; ESRD, end-stage renal disease; HIV, human immunodeficiency virus; LOS, length of stay; NF, nosocomial fever. 
The median duration from admission to the onset of NF was 12 (4.7-21.2) days. The mean body temperature at the onset was $38.96 \pm 0.55^{\circ} \mathrm{C}$. During the onset of NF, 77 $(89.5 \%)$ subjects were already receiving antimicrobial treatments, but only $39(50.6 \%)$ received appropriate antimicrobial therapy. The median length of stay (LOS) was 40 (24-62) days, and 23 (26.7\%) subjects had fatal outcomes.

Chest radiography identified the etiology of NF in 27 (31.4\%) of subjects, particularly in subjects who were suspected hospital associated pneumonia (Table 2). Blood culture was performed in $84(97.7 \%)$, but only $9(10.7 \%)$ yielded positive results. Cultures of respiratory samples from 53 subjects and of urine samples from 60 subjects were conducted, only $20(37.7 \%)$ of respiratory cultures, and $12(20.0 \%)$ of urine cultures were able to identify the causative pathogen. Imaging studies - computed tomography 18 (20.9\%) and ultrasonography $14(16.3 \%)$ were less frequently conducted diagnostic investigations, but both imaging modalities provided a higher positive diagnostic finding. A varying number of subjects underwent additional blood testing for aiding diagnosis cause of NF, such as antinuclear antibody (ANA) titer (17), lactate dehydrogenase (LDH) level (14), procalcitonin (PCT) level (20), and C-reactive protein (CRP) level (8). The additional blood testing exhibited a substantial difference in the rate of positive diagnostic findings (Table 2). Tissue biopsy was performed in 12 subjects and identified the causes of NF in $6(50 \%)$ cases.

NF caused by nosocomial infections was found in 57 $(66.3 \%)$ of subjects, including hospital-associated pneumonia $(27,47.4 \%)$, urinary tract infection $(13,22.8 \%)$, bacteremia $(5,8.8 \%)$, and gastrointestinal infection (5, $8.8 \%$ ) (Table 3). Fifty-one (59.3\%) subjects had multidrug resistant (MDR) gram-negative bacilli, such as ESBL-producing Enterobacteriaceae (Klebsiella pneumoniae and Escherichia coli), Acinetobacter baumannii, and Pseudomonas aeruginosa while only two subjects had non-MDR gram-negative bacilli identified - one from Proteus mirabilis and another from Shewanella

Table 2 Laboratory and Diagnostic Investigations to Identify the Etiologies of Nosocomial Fever

\begin{tabular}{|c|c|c|}
\hline Laboratory Testing and Diagnostic Investigations & Total, $\mathbf{n}$ & $\begin{array}{l}\text { Positive Findings Related } \\
\text { to the Identified } \\
\text { Etiology, } \mathbf{n}(\%)^{\mathrm{a}}\end{array}$ \\
\hline Complete blood count & 86 & \\
\hline Mean hemoglobin (SD), g/dL & $9.52(1.79)$ & \\
\hline Median white blood count (P25-P75), $\times 10^{3}$ cells $/ \mathrm{mm}^{3}$ & $10.31(7.39-13.83)$ & \\
\hline Median platelet count (P25-P75), $\times 10^{3}$ cells $/ \mathrm{mm}^{3}$ & $199(124-272)$ & \\
\hline \multicolumn{3}{|l|}{ Radiographic study } \\
\hline Chest radiography & 86 & $27(31.4)$ \\
\hline Computed tomography & 18 & $13(72.2)$ \\
\hline Ultrasonography & 14 & $7(50.0)$ \\
\hline \multicolumn{3}{|l|}{ Cultures } \\
\hline Blood culture & 84 & $9(10.7)$ \\
\hline Respiratory sample culture & 53 & $20(37.7)$ \\
\hline Urine culture & 60 & $12(20.0)$ \\
\hline \multicolumn{3}{|l|}{ Other investigations } \\
\hline Blood antinuclear antibody (ANA) testing & 17 & $\mathrm{I}(5.9)^{\mathrm{b}}$ \\
\hline Blood lactate dehydrogenase (LDH) testing & 14 & $2(14.3)^{c}$ \\
\hline Blood procalcitonin (PCT) testing & 20 & $7(35)^{d}$ \\
\hline Blood C-reactive protein (CRP) testing & 8 & $6(75)^{e}$ \\
\hline Tissue biopsy and diagnosis & 12 & $6(50.0)^{f}$ \\
\hline
\end{tabular}

Notes: ${ }^{a} \mathrm{t}$ is defined as the rate of positive results from laboratory and diagnostic investigations that gave rise to establish the etiology of nosocomial fever or the results could aid the etiology identification. ${ }^{b}$ One subject developed an exacerbation of systemic lupus erythematosus and having a highly elevated ANA titer. ${ }^{\mathrm{C}}$ One subject with diagnosis of Burkitt lymphoma (LDH level of $1693 \mathrm{U} / \mathrm{L}$ ) and one subject with diagnosis of peripheral T cell lymphoma (LDH level of $2500 \mathrm{U} / \mathrm{L}$ ). ${ }^{\text {dPCT levels ranged from } 0.55-}$

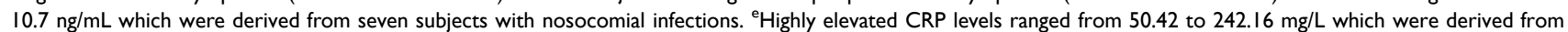
various etiologies, such as drug fever (2), bacteremia (I), pneumonia (I), spondylodiscitis (I) and peripheral T cell lymphoma (I). ${ }^{\mathrm{V}}$ arious etiologies identified, such as lymphoma (3), hematoma (I), adrenal hemorrhage (I), and cerebral thrombosis (I). 
Table 3 Infectious and Non-Infectious Etiologies Identified in Subjects with Nosocomial Fever

\begin{tabular}{|l|l|}
\hline Etiology & Total (n = 86) \\
\hline Infectious etiology (\%) & $\mathrm{n}=57$ \\
Hospital-associated pneumonia & $27(47.4)$ \\
Urinary tract infection & $13(22.8)$ \\
Bacteremia & $5(8.8)$ \\
Gastrointestinal tract infection & $5(8.8)$ \\
Skin and soft tissue infection & $2(3.5)$ \\
Upper respiratory tract infection & $2(3.5)$ \\
Central nervous system infection & $2(3.5)$ \\
Lumbar spondylodiscitis & $\mathrm{I}(1.7)$ \\
\hline Non-infectious etiology (\%) & $\mathrm{n}=28$ \\
Malignancy & $5(17.8)$ \\
Hematoma & \\
Procedure-related & \\
Drug-related & $4(14.3)$ \\
Crystal-induced arthritis & $4(14.3)$ \\
Cerebral thrombosis & $3(10.7)$ \\
Myocardial infarction & $2(7.1)$ \\
Adrenal crisis & $\mathrm{I}(3.6)$ \\
Bowel ischemia & $\mathrm{I}(3.6)$ \\
Exacerbation of systemic lupus erythematosus & $\mathrm{I}(3.6)$ \\
Others & $\mathrm{I}(3.6)$ \\
Unknown & $\mathrm{I}(3.6)$ \\
\hline
\end{tabular}

Notes: a Lymphoma $(n=4)$, neuroendocrine tumor $(n=1)$. ${ }^{\text {Intramuscular sites of }}$ extremities $(n=3)$ and intra-abdominal cavity $(n=1)$. ${ }^{c}$ Mechanical thrombectomy $(n=2)$, percutaneous biliary drainage $(n=2)$.

putrefaciens (Supplementary Table 2). Of 28 (32.5\%) cases of non-infectious NF, five $(17.8 \%)$ were malignancy (lymphoma 4, neuroendocrine tumor 1), four (14.3\%) were large hematoma, four (14.3\%) were procedure-related, and three $(10.7 \%)$ were drug-related (Table 3). The large hematomas were located in the intramuscular sites of the extremities (3) and the intraabdominal cavity (1). One case was classified as unknown etiology because the subject was referred to another hospital for further management.

Most clinical characteristics were broadly similar in both the infectious etiology and non-infectious etiology groups (Table 4). However, acute physiology and chronic health evaluation II (APACHE II) and sequential organ failure assessment (SOFA) scores at the NF onset were significantly higher in the infectious group. The day of onset of NF after hospitalization and body temperature at the onset, including the median duration of etiology identification (5 days) were not significantly different. However, subjects in the infectious group had significantly greater rates of pertinent symptoms and physical findings $(\mathrm{p}<0.01)$, blood urea nitrogen $(\mathrm{BUN})(\mathrm{p}=0.03)$ and creatinine $(\mathrm{p}=0.05)$ levels, abnormal chest radiography $(\mathrm{p}<0.01)$, and positive blood culture $(\mathrm{p}=0.05)$. Antimicrobial treatment was prescribed in $19(67.9 \%)$ of non-infectious group and in $100 \%$ of subjects in the infectious group $(\mathrm{p}<0.01)$, while only three $(15.8 \%)$ of the non-infectious group and $36(63.2 \%)$ of the infectious group had appropriate indications for antimicrobial treatment $(\mathrm{p}<0.01)$. Eighteen subjects underwent medical procedures following the diagnosis of NF. Subjects with non-infectious NF were more likely to undergo tissue biopsy $(\mathrm{p}<0.01)$. Length of stay ( 30 days vs 43 days; $\mathrm{p}=0.35)$ and mortality rate $(17.9 \%$ vs $31.6 \% ; \mathrm{p}=0.16)$ were lower in the non-infectious NF group.

\section{Factors Associated with in-Hospital Mortality}

Baseline demographic data and clinical findings, comorbidities, etiologies of NF, and clinical management were comparable between survival and non-survival groups (Supplementary Table 3). However, the non-survival group had significantly higher APACHE II (15.87 vs 13.02, $\mathrm{p}=0.02$ ) and SOFA scores ( 5 vs $2, \mathrm{p}=0.001)$. The non-survival group also had a longer time before the onset of NF (17 days vs 8 days, $\mathrm{p}=0.05$ ), a greater rate of pertinent symptoms and physical findings $(86.9 \%$ vs $61.9 \%, \mathrm{p}=0.03)$, more elevated BUN level $(46.20 \mathrm{mg} / \mathrm{dL}$ vs $26.60 \mathrm{mg} / \mathrm{dL}, \mathrm{p}=0.02$ ), and a greater rate of abnormal chest radiography $(52.2 \%$ vs $27.0 \%, \mathrm{p}=0.03)$. In addition, a significantly longer LOS was observed in the nonsurvival group (61 days vs 36 days, $\mathrm{p}=0.02)$. Multivariate analysis revealed that end-stage renal disease (ESRD) [odd ratio (OR) 19.49 (1.77-214.18), $\mathrm{p}=0.015$ ], SOFA scores $>6$ [OR 5.18 (1.04-25.90), $\mathrm{p}=0.045$ ], and abnormal chest radiography [OR 3.46 (1.16-10.29), $\mathrm{p}=0.026]$ were significantly associated with a fatal outcome (Table 5).

\section{Discussion}

We were able to establish the etiology of nosocomial fever in $98.8 \%$ of subjects, a higher rate of identification than in some previous reports $(80-89 \%))^{1,5,9-11}$ Our prospective study design allowed us to follow our subjects until they were discharged. Together with careful clinical evaluation, additional laboratory testing and diagnostic investigations, we were able to identify the etiology in all but a single case. The time from admission to the onset of NF was 12 
Table 4 Comparison of Subjects with Nosocomial Fever Categorized by Infectious and Non-Infectious Etiologies

\begin{tabular}{|c|c|c|c|}
\hline & $\begin{array}{l}\text { Infectious Etiology } \\
\text { Group }(n=57)\end{array}$ & $\begin{array}{l}\text { Non-Infectious Etiology } \\
\text { Group }(n=28)\end{array}$ & $p$-value \\
\hline Male (\%) & $25(43.9)$ & $10(35.7)$ & 0.40 \\
\hline Mean age (SD), years & $69.95(15.7)$ & $68.00(20.8)$ & 0.66 \\
\hline Mean BMI (SD), kg/m² & $21.66(5.5)$ & $20.55(3.4)$ & 0.25 \\
\hline \multicolumn{4}{|l|}{ Underlying disease (\%) } \\
\hline Hypertension & $36(63.2)$ & $15(53.6)$ & 0.31 \\
\hline Dyslipidemia & $20(35.1)$ & II (39.3) & 0.79 \\
\hline Diabetes mellitus & $20(35.1)$ & $10(35.7)$ & 0.96 \\
\hline CKD or ESRD & $16(28.1)$ & $6(21.4)$ & 0.51 \\
\hline Cerebrovascular disease & $12(21.1)$ & $9(32.1)$ & 0.31 \\
\hline Coronary arterial disease or arrhythmia & $9(15.8)$ & $7(25.0)$ & 0.31 \\
\hline Autoimmune diseases & $8(14.0)$ & $5(17.9)$ & 0.76 \\
\hline Asthma or COPD & $5(8.8)$ & $2(7.1)$ & 1.00 \\
\hline Chronic liver disease & $4(7.0)$ & $3(10.7)$ & 0.68 \\
\hline HIV infection & $0(0 \%)$ & I (3.6) & \\
\hline Organ transplant recipient & $\mathrm{I}(\mathrm{I} .8)$ & $0(0 \%)$ & \\
\hline Immunocompromised state (\%) & $12(21.1)$ & $6(21.4)$ & 0.97 \\
\hline Living in dependence status (\%) & $23(40.4)$ & $13(46.4)$ & 0.69 \\
\hline Median Charlson Comorbidity Index (P25-P75) & $5(3-6)$ & $5(3-6)$ & 0.77 \\
\hline Mean APACHE II (SD) & $14.70(5.18)$ & II.97 (4.40) & 0.02 \\
\hline Median SOFA score (P25-75) & $4(2-4)$ & $2(0-3)$ & $<0.01$ \\
\hline \multicolumn{4}{|l|}{ Medical procedure prior to NF diagnosis (\%) } \\
\hline Urinary catheterization & $41(71.9)$ & $18(64.3)$ & 0.35 \\
\hline Nasogastric tubing & $33(57.9)$ & $14(50)$ & 0.40 \\
\hline Endotracheal intubation & $17(29.8)$ & $8(28.6)$ & 0.83 \\
\hline Intravascular catheterization & $9(15.8)$ & $4(14.3)$ & 1.00 \\
\hline Percutaneous drainage & $\mathrm{I}(\mathrm{I} .8)$ & I (3.6) & 1.00 \\
\hline Recent surgery (\%) & $8(14.0)$ & $6(21.4)$ & 0.54 \\
\hline \multicolumn{4}{|l|}{ Clinical characteristics of nosocomial fever } \\
\hline Median onset (P25-P75), days & $13(5-24)$ & $8(6-20)$ & 0.35 \\
\hline Mean body temperature (SD), ${ }^{\circ} \mathrm{C}$ & $38.96(0.6)$ & $38.97(0.5)$ & 0.53 \\
\hline Median duration of identifying etiology (P25-P75), days & $5(4-7)$ & $5(3-10)$ & 0.78 \\
\hline Pertinent symptoms and physical findings at the onset (\%) & $47(82.5)$ & $12(42.9)$ & $<0.01$ \\
\hline \multicolumn{4}{|l|}{ Laboratory testing and investigations } \\
\hline Mean Hb level (SD), g/dL & $9.54(1.74)$ & $9.48(1.91)$ & 0.89 \\
\hline Median WBC count (P25-P75), $\times 10^{3}$ cells $/ \mathrm{mm}^{3}$ & $10.17(7.82-14.3)$ & $10.39(6.07-12.81)$ & 0.38 \\
\hline Median platelet count $(\mathrm{P} 25-75), \times 10^{3}$ cells $/ \mathrm{mm}^{3}$ & $202(|3|-270)$ & $174(109-259)$ & 0.45 \\
\hline Median BUN level (P25-75), mg/dL & $37.30(24.60-56.50)$ & $21.10(13.70-51.45)$ & 0.03 \\
\hline Median $\mathrm{Cr}$ level (P25-75), mg/dL & $1.41(0.75-2.11)$ & $0.97(0.50-1.99)$ & 0.05 \\
\hline Abnormal chest radiography (\%) & $26(45.6)$ & I (3.6) & $<0.01$ \\
\hline Abnormal US or CT findings (\%) & $13(22.8)$ & $7(25)$ & 0.68 \\
\hline Blood cultures positive (\%) & $9(15.8)$ & $0(0 \%)$ & 0.05 \\
\hline Sputum cultures positive (\%) & $20(35.1)$ & $8(28.6)$ & 0.23 \\
\hline Urine cultures positive (\%) & $12(21.1)$ & $3(10.7)$ & 0.09 \\
\hline \multicolumn{4}{|l|}{ Managements (\%) } \\
\hline Receiving antimicrobial treatment & $57(100)$ & $19(67.9)$ & $<0.01$ \\
\hline Appropriate antimicrobial treatment & $36(63.2)$ & $3(15.8)$ & $<0.01$ \\
\hline Surgical treatment & $3(5.3)$ & I (3.6) & 1.00 \\
\hline Blood transfusion therapy & $16(28.1)$ & II (39.3) & 0.35 \\
\hline
\end{tabular}

(Continued) 
Table 4 (Continued).

\begin{tabular}{|l|l|l|l|}
\hline & $\begin{array}{l}\text { Infectious Etiology } \\
\text { Group (n= 57) }\end{array}$ & $\begin{array}{l}\text { Non-Infectious Etiology } \\
\text { Group (n = 28) }\end{array}$ & $p$-value \\
\hline Medical procedure post NF diagnosis & & & 0.01 \\
Tissue Diagnosis & $3(5.3)$ & $9(32.1)$ & 0.26 \\
Lumbar puncture & $1(1.8)$ & $2(7.1)$ & 1.00 \\
Endoscopy & $2(3.5)$ & $30(24-5 I)$ & 0.35 \\
Median LOS (P25-P75), days & $43(24-65)$ & & 0.16 \\
\hline Outcome (\%) & & $5(17.9)$ & \\
Death & $18(31.6)$ & \\
\hline
\end{tabular}

Abbreviations: APACHE II, acute physiology and chronic health evaluation II; BMI, body mass index; BUN, blood urea nitrogen; CKD, chronic kidney disease; COPD, chronic obstructive pulmonary disease; Cr, creatinine; CT, computed tomography; ESRD, end-stage renal disease; Hb, hemoglobin; HIV, human immunodeficiency virus; LOS, length of stay; NF, nosocomial fever; SOFA, sequential organ failure assessment; US, ultrasonography; WBC, white blood cell.

Table 5 Univariate and Multivariate Analysis of Factors Associated with in-Hospital Mortality in Subjects with Nosocomial Fever

\begin{tabular}{|c|c|c|c|c|}
\hline \multirow[t]{2}{*}{ Variables } & \multicolumn{2}{|l|}{ Univariate } & \multicolumn{2}{|l|}{ Multivariate } \\
\hline & OR $(95 \% \mathrm{Cl})$ & p-value & OR $(95 \% \mathrm{CI})$ & $p$-value \\
\hline End-stage renal disease & $9.30(0.92-94.50)$ & 0.059 & $19.49(1.77-2 \mid 4.18)$ & 0.015 \\
\hline APACHE II score & $1.12(1.01-1.24)$ & 0.026 & & \\
\hline SOFA $>6$ & $5.56(1.21-25.53)$ & 0.028 & $5.18(1.04-25.90)$ & 0.045 \\
\hline Pertinent symptoms and physical findings at the onset (\%) & $4.10(1.10-15.29)$ & 0.035 & & \\
\hline Abnormal chest radiography & $2.95(1.10-7.94)$ & 0.032 & $3.46(1.16-10.29)$ & 0.026 \\
\hline
\end{tabular}

Abbreviations: APACHE II, acute physiology and chronic health evaluation II; SOFA, sequential organ failure assessment.

days, which is comparable to previous studies that reported approximately $7-13$ days. ${ }^{1,9}$ Nosocomial infections were the most common cause of NF, consistent with other studies where the frequency of infectious etiology ranged from 56-77\%, with hospital-associated pneumonia and urinary tract infection representing the majority of cases. $^{1,5,9,11,12}$

We were also able to identify a variety of noninfectious NF caused by malignancy, large hematoma, procedure-related fever, drug-related fever, and crystalinduced arthritis. Uncommon causes of non-infectious NF were also identified, such as cerebral thrombosis, myocardial infarction, adrenal crisis, bowel ischemia, and exacerbation of systemic lupus erythematosus. Further blood testing performed in the current study could little aid diagnosis the cause of NF. An increased CRP level provided a high rate of positive diagnostic findings (75\%) but it could not distinguish between the infectious and non-infectious causes of NF. Meanwhile, imaging studies (CT or US) or tissue biopsy can be useful to identify the cause of NF. Thus, when a hospitalized patient develops a new onset of fever with no obvious source of infection, an integrated approach using comprehensive evaluation and investigations will help identify other causes of nosocomial fever. A 1-year study by Trivalle et al reported that advanced age, multiple comorbidities, and immunocompromised state are associated with developing of NF, but did not compare between infectious and non-infectious etiologies. $^{5}$ Leukocytosis may be associated with infectious etiology, ${ }^{1}$ but we did not find an association between white blood cell count and the etiology of $\mathrm{NF}^{12,16}$ Laboratory testing, such as culture from blood and chest radiography can also provide information that can separate infectious and non-infectious etiologies. ${ }^{5,16}$ The current study demonstrated a review of the patient's medical history and symptoms, and careful physical examination are the first steps of an integrated approach. In approximately $80 \%$ of infectious causes the pertinent symptoms and physical findings were present, while only $40 \%$ of noninfectious causes would have such symptoms and physical findings at the onset.

Antimicrobials were prescribed to $89.5 \%$ of subjects, but the appropriate indication for antimicrobial treatment was present in only $50.6 \%$ of these cases. The presence of high-grade fever cannot reliably distinguish between infectious NF and non-infectious NF. Due to concerns about 
high body temperature, physicians usually prescribe antimicrobial treatment while waiting for laboratory results, and this often results in several days of unnecessary treatment in non-infectious NF subjects. Other studies have also reported unnecessary antibiotic prescriptions, ranging from $8 \%$ to $58 \%$ in subjects with NF. ${ }^{5,9,16}$ However, empirical antimicrobial treatment might be reasonable in subjects with suspected infectious NF, particularly in subjects with high APACHE II and/or SOFA scores at the onset.

The mean length of hospital stay in subjects with NF was 40 days, the same as a previous study in our hospital, $^{12}$ and the overall mortality rate was $26.7 \%$. However, several previous studies reported a shorter length of hospital stay (13-20 days) $)^{1,2,5,16}$ and a lower mortality rate $(9-16 \%))^{2,4,5,16}$ This can be explained by differences in comorbidity, immunity, living status, and severity in our subjects and those from other studies. Interestingly, subjects with an infectious etiology were not more likely to have a fatal outcome $(\mathrm{p}=0.16)$, but several independent factors were strongly associated with mortality in subjects with NF, such as end-stage renal disease, SOFA score $>6$, and abnormal findings from chest radiography. These findings should prompt physicians to seek prompt diagnosis and careful management to prevent a fatal outcome.

Our study had some limitations. Our sample size was small, which affected our ability to detect a statistical difference in some clinical variables. Some laboratory testing and diagnostic investigations did not apply to all subjects, the type of investigation was selected based on case-by-case evaluation. A comprehensive diagnostic investigation to identify the etiology of nosocomial fever may be limited due to the individual treatment plan, goal of treatment, and the patient's living status. In fourteen subjects, the patient care team decided to give the patient supportive care rather than conduct an aggressive clinical investigation to determine the cause of the fever. However, we did not include these fourteen subjects for study analysis. Finally, we studied subjects admitted to the general medicine ward only, and so our findings may not apply to other types of patients.

\section{Conclusion}

In conclusion, nosocomial infections and non-infectious causes such as malignancy and hematoma were the leading causes of NF in hospitalized patients. Comprehensive clinical evaluation and diagnostic investigations are warranted to identify the etiology of NF and to prevent prolonged hospital stays and reduce in-hospital mortality. NF occurring in hospitalized patients with ESRD, having SOFA scores $>6$, and abnormal chest radiography should receive a prompt evaluation and management to prevent a fatal outcome. To inform preventive measures needed to reduce the burden of NF, a large prospective study in multiple patient populations is needed.

\section{Abbreviations}

ANA, antinuclear antibody; APACHE II, acute physiology and chronic health evaluation II; BUN, blood urea nitrogen; CDC, Centers for Disease Control and Prevention; CRP, C-reactive protein; CT, computed tomography; ESRD, end-stage renal disease; HIV, human immunodeficiency virus; LDH, lactate dehydrogenase; LOS, length of stay; MDR, multidrug resistant; NF, nosocomial fever; NHSN, National Healthcare Safety Network; PCT, procalcitonin; SOFA, sequential organ failure assessment; US, ultrasonography.

\section{Data Sharing Statement}

Primary datasets from this study will be available from the corresponding author upon request.

\section{Ethics Approval and Informed Consent}

The study was approved by the Scientific Ethics Committee of the Siriraj Institutional Review Board (Approval No. Si 622/2019). This study was conducted in accordance with the Declaration of Helsinki. For an organ transplant recipient, the organ was donated voluntarily with written informed consent, and the study was conducted in accordance with the Declaration of Istanbul. Informed consent was obtained from all subjects involved in the study.

\section{Acknowledgments}

The authors gratefully acknowledge the patient care teams and nursing staff of the Department of Medicine, Faculty of Medicine, Siriraj Hospital, Mahidol University, for their assistance with the clinical data collection and evaluation, and their collaboration in this study.

\section{Author Contributions}

All authors made substantial contributions to conception and design, acquisition of data, or analysis and 
interpretation of data; took part in drafting the article or revising it critically for important intellectual content; gave final approval of the version to be published; and agree to be accountable for all aspects of the work.

\section{Funding}

This research did not receive any specific grant from funding agencies in the public, commercial, or not-forprofit sectors.

\section{Disclosure}

The authors report no conflicts of interest in this work.

\section{References}

1. Arbo MJ, Fine MJ, Hanusa BH, Sefcik T, Kapoor WN. Fever of nosocomial origin: etiology, risk factors, and outcomes. Am J Med. 1993;95(5):505-512. doi:10.1016/0002-9343(93)90333-K

2. Bor DH, Makadon HJ, Friedland G, Dasse P, Komaroff AL, Aronson MD. Fever in hospitalized medical patients: characteristics and significance. J Gen Intern Med. 1988;3(2):119-125. doi:10.1007/ BF02596115

3. Kaul DR, Flanders SA, Beck JM, Saint S. Brief report: incidence, etiology, risk factors, and outcome of hospital-acquired fever: a systematic, evidence-based review. J Gen Intern Med. 2006;21 (11):1184-1187. doi:10.1111/j.1525-1497.2006.00566.x

4. Moon SY, Park KH, Lee MS, Son JS. Hospital-acquired fever in oriental medical hospitals. BMC Health Serv Res. 2018;18(1):88. doi:10.1186/s12913-018-2896-1

5. Trivalle C, Chassagne $\mathrm{P}$, Bouaniche $\mathrm{M}$, et al. Nosocomial febrile illness in the elderly: frequency, causes, and risk factors. Arch Intern Med. 1998;158(14):1560-1565. doi:10.1001/archinte.158.14.1560

6. Walter EJ, Hanna-Jumma S, Carraretto M, Forni L. The pathophysiological basis and consequences of fever. Crit Care. 2016;20(1):200. doi:10.1186/s13054-016-1375-5
7. Allegranzi B, Bagheri Nejad S, Combescure C, et al. Burden of endemic health-care-associated infection in developing countries: systematic review and meta-analysis. Lancet. 2011;377 (9761):228-241. doi:10.1016/S0140-6736(10)61458-4

8. Gajdács M, Bátori Z, Ábrók M, Lázár A, Burián K. Characterization of resistance in gram-negative urinary isolates using existing and novel indicators of clinical relevance: a 10-year data analysis. Life. 2020;10(2):16.

9. Filice GA, Weiler MD, Hughes RA, Gerding DN. Nosocomial febrile illnesses in patients on an internal medicine service. Arch Intern Med. 1989;149(2):319-324. doi:10.1001/archinte.1989.00390020051011

10. McGowan JE Jr, Rose RC, Jacobs NF, Schaberg DR, Haley RW. Fever in hospitalized patients. With special reference to the medical service. Am J Med. 1987;82(3Spec No):580-586. doi:10.1016/00029343(87)90103-3

11. Ueno K, Hayashi J, Yamaga S, et al. Febrile episodes in elderly inpatients- a one year survey to determine the causes of fever in hospital. Kansenshogaku Zasshi. 1998;72(5):493-498. doi:10.11150/ kansenshogakuzasshi1970.72.493

12. Rutjanawech S, Koomanachai P. Nosocomial febrile illness in internal medicine hospitalized patients, Siriraj Hospital. J Infect Dis Antimicrob Agents. 2020;37:111-122.

13. O'Grady NP, Barie PS, Bartlett JG, et al. Guidelines for evaluation of new fever in critically ill adult patients: 2008 update from the American college of critical care medicine and the infectious diseases society of America. Crit Care Med. 2008;36(4):1330-1349. doi:10.1097/CCM.0b013e318169eda9

14. Auesomwang C, Suwannawiboon B, Chayakulkeeree M. Changes in etiologic microorganisms in Thai patients with chemotherapy-induced neutropenia and fever. $J$ Med Assoc Thai. 2018;101:173-180.

15. Horan TC, Andrus M, Dudeck MA. CDC/NHSN surveillance definition of health care-associated infection and criteria for specific types of infections in the acute care setting. Am J Infect Control. 2008;36 (5):309-332. doi:10.1016/j.ajic.2008.03.002

16. Eroles Vega G, Fernández Pérez C, Del Valle Loarte P, et al. Fever in patients of a service of internal medicine: prospective study in 204 cases. An Med Interna. 2006;23(2):56-61.
Infection and Drug Resistance

\section{Publish your work in this journal}

Infection and Drug Resistance is an international, peer-reviewed openaccess journal that focuses on the optimal treatment of infection (bacterial, fungal and viral) and the development and institution of preventive strategies to minimize the development and spread of resistance. The journal is specifically concerned with the epidemiology of antibiotic resistance and the mechanisms of resistance development and diffusion in both hospitals and the community. The manuscript management system is completely online and includes a very quick and fair peerreview system, which is all easy to use. Visit http://www.dovepress.com/ testimonials.php to read real quotes from published authors. 